\section{Improvement in patients' quality-of- life following strabismus surgery: evaluation of postoperative outcomes using the Adult Strabismus 20 (AS-20) score}

P Glasman, R Cheeseman, V Wong, J Young and JM Durnian in the score with larger changes in deviation size. The smaller the postoperative angle, the larger the final AS-20 score.

Eye (2013) 27, 1249-1253; doi:10.1038/eye.2013.174; published online 9 August 2013

Keywords: strabismus; AS-20; quality-of-life; postoperative outcomes

\section{Introduction}

Adult strabismus is common, affecting up to $4 \%$ of the population. ${ }^{1}$ Its most noticeable effect is on the facial appearance of affected individuals; however, the widespread belief that corrective squint surgery is a purely cosmetic procedure is incorrect. First, it seeks to restore the normality disrupted by a disease process rather than gild or glamorise. Second, strabismic adults have been shown to have social and psychological issues directly related to their condition, which impact all aspects of their lives: education, social relationships, employment prospects, and self-esteem. ${ }^{2-7}$ One study has shown that manifest strabismus has a direct relationship with mental health issues, which occur more commonly when compared with controls. ${ }^{8}$ A review of the psychosocial issues associated with squint concluded thus, 'There is overwhelming evidence describing these negative effects on patients. ${ }^{\prime 9}$

Until recently, documentation of the overall impact of strabismus was challenging, being assessed using generic or locally produced
St Paul's Eye Unit, Royal Liverpool University Hospital, Liverpool, UK

Correspondence: JM Durnian, St Paul's Eye Unit, Royal Liverpool University Hospital, Prescot Street, Liverpool L7 8XP, UK.

Tel: + 44 (0)151 706 3992; Fax: + 44 (0)151 7065905 . E-mail: jondurnian@ me.com

Received: 21 January 2013 Accepted in revised form: 4 July 2013

Published online: 9 August 2013 
questionnaires. ${ }^{9}$ Although this undoubtedly provided useful information, they were limited by the absence of condition-specific questions or lack of validation. These issues were addressed by the development of the strabismus-specific quality-of-life questionnaire (QoL), the Adult Strabismus 20 (AS-20), which assesses both the functional and psychosocial aspects of strabismus. ${ }^{10} \mathrm{It}$ has been shown to be superior in comparisons with existing QoL assessment tools, ${ }^{7,11}$ with a high retest reliability. ${ }^{12}$

The AS-20 provides a basis for assessing the holistic effects of squint surgery by comparing pre- and postoperative scores and relating these to the objective surgical success of the procedure.

The aim of our project was threefold: (1) to assess any change in the AS-20 scores with strabismus surgeryboth the total score and of its subscales; (2) to compare this change with the surgical results; and (3) to examine the preoperative variables (gender, size of preoperative deviation, and direction of deviation) and surgical variables (change in deviation size and size of postoperative deviation) to see if there is any relationship to the change in AS-20 scores.

\section{Materials and methods}

\section{The AS-20}

The AS-20 is a freely available QoL questionnaire ${ }^{10}$ developed specifically for strabismic adults. Development started with patients' interviews, which provided 181 questions derived from their unique statements. These were then distilled down to a 20-point questionnaire with each question being a good discriminator. Each question uses a five-point Likert scoring scale: 'never' (score 100); 'rarely' (score 75); 'sometimes' (score 50); 'often' (score 25); and 'always' (score 0 ). The overall score is the mean of all the questions answered, with a best-possible score of 100 and worst, 0 . The threshold score for a normal, nonstrabismic adult is 84 . The AS-20 was developed with two subscales: one concerned with the psychosocial aspects of strabismus (AS-20py) and one with its functional aspects (AS-20fn).

The test-retest reliability of the AS-20 is good, indicating its potential for its use in assessing changes in strabismus over a long time period. The AS-20 shows a much greater sensitivity to the effects of strabismus than the widely used 25-item National Eye Institute Visual Function Questionnaire ${ }^{11}$ and correlates well to the Derriford Appearance Scale 59 research tool, indicating that the AS-20 is not influenced by other bodily factors that can impinge on patients' perception of their own appearance. $^{7}$
We report a prospective cohort study of consecutive adult patients undergoing squint surgery between August 2010 and February 2012. Patients who were listed for strabismus surgery were asked to complete the AS-20 at their pre-assessment clinic visit by the specialist orthoptist (JY or VW), following their orthoptic assessment. Patients were then asked to complete another AS-20 at their final postoperative visit, again by the orthoptist. On both occasions the patient was left alone to complete the assessment to try and get the most honest responses. The data were not looked at in the presence of the patient. All surgeries were performed by or under the supervision of the senior author (JMD).

Full pre- and postoperative orthoptic measurements were recorded. Pre- and postoperative manifest deviations were compared with the respective AS-20 scores and subscales. The patients' age, sex; size of deviation, and direction of deviation was recorded preoperatively. In order to incorporate any vertical deviations into the analysis, manifest horizontal ( $\left.\mathrm{dev}_{\mathrm{h}}\right)$ and vertical $\left(\mathrm{dev}_{\mathrm{v}}\right)$ deviations were treated as vectors and their magnitudes were combined $(|\operatorname{dev}|)$ as per mathematical convention: $|\mathrm{dev}|=\sqrt{ }\left(\operatorname{dev}_{\mathrm{h}}{ }^{2}+\operatorname{dev}_{\mathrm{v}}{ }^{2}\right)$.

The AS-20 scores were analysed as quantitative, discrete data. Pre- and postoperative measurements were compared using the Wilcoxon signed-rank test. Statistical significance was counted as $P<0.05$ throughout. All statistics were performed using MedCalc version 9.3.0.0 (MedCalc Software, Ostend, Belgium).

Ethical approval was sought but deemed not necessary for this service evaluation project by the Research and Development Department of the Royal Liverpool University Hospital. The research was performed in compliance with the tenets of the Declaration of Helsinki.

Our exclusion criteria for this study were patients under the age of 16 , lack of capacity to consent verbally to the test or retain the information required to perform it, and patients who had other ocular or facial abnormalities, such as thyroid eye disease or VII palsy.

\section{Results}

We identified 86 patients with complete pre-, peri-, and postoperative data. The mean age of the patients was 41 years (range 17-76) and 47\% (40) of them were female. The mean follow-up between date of surgery and final AS-20 score was 91 days (range 12-362, SD 73.7).

In all, $60 \%$ (52) of cases had a manifest exo-deviation and $50 \%$ (43) of the cases had a manifest vertical deviation preoperatively. The median horizontal deviation was 30 prism diopters (PD) (95\% CI: 20-35) and where a vertical deviation was present, median vertical deviation was 7 PD (95\% CI: 5-10). Combining the deviations gave a median magnitude of total deviation of 30.5 PD (95\% CI: 23.5-35). 
The median total AS-20 score preoperatively was 45 (95\% CI: 38-48.2). Of the subscales, the AS-20py had a median score of 38.8 (95\% CI: 31.1-50) and the AS-20fn had a median score of 46.2 (95\% CI: 40-51.4). The preoperative scores for males and females, and esotropes and exotropes can be seen in Table 1.

Mann-Whitney analysis of the preoperative subgroups shows no difference in the AS-20 scores between esotropes and exotropes $(Z=0.415, P=0.6782$ for total AS-20 score). Females tend to score more negatively on the AS-20 than males $(Z=2.204, P=0.0275)$ with the AS-20fn subscale also being significantly lower ( $Z=2.135, P=0.0327)$. AS-20py shows no significant difference $(Z=1.724, P=0.0846)$.

Postoperatively, the median magnitude of horizontal deviation was 6 PD (95\% CI: 4-8) and vertical deviation (where present initially) was 5 PD (95\% CI: 4-6).

The median total deviation postoperatively was $8 \mathrm{PD}$ (95\% CI: 6.3-10.7). The postoperative deviations were significantly less than the preoperative measures ( $Z=7.94, P<0.0001)$.

The median total AS-20 score postoperatively was 73.1 (95\% CI: 66.2-82). The median subscale scores were: AS-20py 77.5 (95\% CI: 67.5-82.5) and AS-20fn 70 (95\% CI: 65-81.4). Pre- and postoperative scores and their comparison can be found in Table 2.

Table 1 Preoperative AS-20 scores and subscores for patient subgroups

\begin{tabular}{lccc}
\hline & $\begin{array}{c}\text { AS-20py } \\
(\text { median })\end{array}$ & $\begin{array}{c}\text { AS-20fn } \\
\text { (median) }\end{array}$ & $\begin{array}{c}\text { Total AS-20 } \\
\text { (median) }\end{array}$ \\
\hline All & 38.8 & 46.3 & 45 \\
patients & 37.5 & 42.5 & 43.1 \\
XT & 45 & 47.5 & 48.8 \\
ET & 47.5 & 51.2 & 47.5 \\
Male & 35 & 40 & 39.4 \\
Female & & & \\
\hline
\end{tabular}

Abbreviations: AS-20fn, functional subscale; AS-20py, psychosocial subscale; ET, esotropia; XT, exotropia.

Table 2 Pre- $v$ s Postoperative AS-20 scores

\begin{tabular}{lccc}
\hline & $\begin{array}{c}\text { Median } \\
\text { preoperative } \\
\text { score }\end{array}$ & $\begin{array}{c}\text { Median } \\
\text { postoperative } \\
\text { score }\end{array}$ & $\begin{array}{c}\text { Wilcoxon comparison } \\
\text { (Z-value, P-value) }\end{array}$ \\
\hline $\begin{array}{l}\text { AS-20 } \\
\text { total }\end{array}$ & 45 & 73.1 & $-7.83, P<0.0001$ \\
$\begin{array}{l}\text { AS- } \\
\text { 20py }\end{array}$ & 38.8 & 77.5 & $-7.487, P<0.0001$ \\
AS- & 46.2 & 70 & $-7.309, P<0.0001$ \\
20fn & & & \\
\hline
\end{tabular}

Abbreviations: AS-20fn, score for the functional subscale; AS-20py, score for the psychosocial subscale; AS-20 total, total score for AS-20 questionnaire.
Looking at the two variables of sex and direction of preoperative, Table 3 shows the results for each variable and the subscales. As is evident, Mann-Whitney analysis showed no significant differences between the postoperative scores within the subgroups.

We correlated the change in manifest deviation with the change in AS-20 (total) score and this showed a correlation of $r=0.291, P=0.0066$.

Using multiple regression with the variables of sex, direction of deviation, and magnitude of total preoperative deviation against the preoperative AS-20 (total) score showed that gender correlates with a lower score $(t=-2.532, P=0.0133)$ whereas deviation size $(t=-0.0984, P=0.1978)$ and deviation direction $(t=0.169, P=0.8666)$ do not.

We applied similar formulae to assess any correlation between sex, postoperative deviation, and change in deviation with: (1) postoperative AS-20 (total) scores and (2) change in AS-20 score. These results can be found in Table 4. Stronger correlations are seen between: (1) smaller postoperative deviation magnitudes and higher AS-20 scores; (2) female gender and a greater increase in AS-20 scores; and (3) larger change in deviation size and a greater increase in AS-20 scores.

\section{Conclusion}

The most important result of our study is that we can now prove the highly significant improvement in patients' QoL that strabismus surgery gives. The postoperative scores are approaching, but do not quite reach, the score that a non-strabismic adult should get on the AS-20. Our postoperative AS-20 score of 73 compares favourably with other authors'. Hatt et al ${ }^{13,14}$ analysed 73 consecutive adults in a cohort study and found an average postoperative score of 78 at 6 weeks, although this did improve to 86 at 1 year. Certainly, the sizeable and statistically significant improvement in scores would support existing evidence that the psychosocial implications of strabismus are improved by surgery. Hatt's data would imply that this process may continue after our mean follow-up of 91 days.

Preoperatively, we found that there was no significant difference in AS-20 scores between esotropes and exotropes, this corresponds well to previous reports with patients self-reporting experiences showing no difference between the difficulties faced between those with esoand exo-deviations. ${ }^{2,5,15,16}$ In contrast in studies with people rating patients with strabismus, esotropes are usually rated worse. ${ }^{17,18}$

Female gender correlated significantly with a lower preoperative AS-20 score. Interestingly, the psychosocial subscale, although showing a difference, is not significantly different to that of males indicating that the 
Table 3 Comparison of postoperative results of the two subgroups

\begin{tabular}{lccc}
\hline & AS-20 total & AS-20py & AS-20fn \\
\hline Male & 76.2 & 73.7 & 72.5 \\
Female & 70.6 & 77.5 & 68.8 \\
Mann-Whitney comparison & $Z=0.02, P=0.9827$ & $Z=0.434, P=0.6645$ & $Z=0.342, P=0.7322$ \\
XT & 75.6 & 70 & 71.2 \\
ET & 67.5 & 72.5 & 70 \\
Mann-Whitney comparison & $Z=0.654, P=0.5132$ & $Z=1.044, P=0.2967$ & $Z=0.284, P=0.7762$ \\
\hline
\end{tabular}

Abbreviations: AS-20fn, functional subscale of AS-20; AS-20py, psychosocial subscale of AS-20; ET, patients with preoperative esotropia; XT, patients with preoperative exotropia.

Table 4 Multiple regression results

\begin{tabular}{lcr}
\hline & Postoperative AS-20 score & Change in AS-20 score \\
\hline Gender & $t=-0.429, P=0.6688$ & $t=1.923, P=0.058^{\mathrm{a}}$ \\
Change in deviation & $t=1.018, P=0.3118$ & $t=2.080, P=0.0406$ \\
Postoperative magnitude of deviation & $t=-1.943, P=0.0555$ & $t=-0.853, P=0.3961$ \\
\hline
\end{tabular}

${ }^{a}$ With females showing more change in score.

females had more functional difficulties with their strabismus than males. This corresponds well with existing studies demonstrating that women with strabismus are rated more negatively by others than their male counterparts. ${ }^{19,20}$

Postoperatively, the AS-20 results show no difference between genders or by preoperative esotropes/exotropes with all groups showing a significant improvement. This result is also bourne out by the close relationship we found between female gender and the increase in AS-20 scores indicating that females seem to get more of an improvement in their QoL scores with strabismus surgery than males.

We report a close relationship between change in deviation size and change in AS-20 score but no similar relationships to the final AS-20 score. There is also a close relationship between final postoperative angle and final AS-20 score but not change in the score. This indicates that you will see higher AS-20 scores with smaller residual angles but you will see a greater improvement in AS-20 score with greater angle change.

Patients in our cohort seemed to score themselves lower preoperatively than patients in the existing literature. A cohort from Minnesota scored a preoperative median of 59,14 which is significantly higher than our score of 45 . Direct comparisons with our study and this one are challenging because of its stratified design, so the reason for this disparity is not clear. Clearly, cultural attitudes or selection bias are possible explanations. Nevertheless, effect size did seem similar (27 vs 24 in the 'surgically successful' Minnesota group).

A recent paper by the authors of the original AS-20 has concluded that the questionnaire may benefit from reducing both the response options to 4 ('never/rarely', 'sometimes', 'often', and 'always') and the number of questions to 18-by deleting the questions regarding depth perception and personal hobbies. ${ }^{21}$ Our study was ongoing when this was published, we feel that the use of the modified AS-20 would not have significantly altered our results.

Bias exists in our study, most obviously by the selection process: all subjects were recruited from the strabismus clinic. Clearly, patients who have been referred to a strabismus service already view their squint as a problem and so may score themselves lower. We would suggest that AS-20 scores in the general, strabismic population would be higher.

In conclusion, we report that strabismus surgery gives a highly significant improvement in, strabismus-specific, QoL scores. Females gain more benefit from surgery and there is a greater improvement in the score with larger changes in deviation size. The smaller the postoperative angle, the larger the final AS-20 score.

\section{Summary}

What was known before

- Strabismus can have a negative impact on a patient's QoL.

- The AS-20 questionnaire quantifies these psychosocial effects.

What this study adds

- Strabismus surgery improves AS-20 scores.

- Change in AS-20 score is correlated with change in angle of squint.

- Women rate themselves more negatively than men. 


\section{Conflict of interest}

The authors declare no conflict of interest.

\section{Disclaimer}

We confirm that our manuscript or any part of it has not been submitted elsewhere for publication. All authors have read the final manuscript and participated sufficiently in the study to take responsibility for it and accept its conclusions.

\section{References}

1 Coats DK, Stager Sr DR, Beauchamp GR, Stager Jr DR, Mazow ML, Paysse EA et al. Reasons for delay of surgical intervention in adult strabismus. Arch Ophthalmol 2005; 123: 497-499.

2 Satterfield D, Keltner JL, Morrison TL. Psychosocial aspects of strabismus study. Arch Ophthalmol 1993; 111: 1100-1105.

3 Burke JP, Leach CM, Davis H. Psychosocial implications of strabismus surgery in adults. J Pediatr Ophthalmol Strabismus 1997; 34: 159-164.

4 Hatt SR, Leske DA, Kirgis PA, Bradley EA, Holmes JM. The effects of strabismus on quality of life in adults. Am J Ophthalmol 2007; 144: 643-647.

5 Nelson BA, Gunton KB, Lasker JN, Nelson LB, Drohan LA. The psychosocial aspects of strabismus in teenagers and adults and the impact of surgical correction. J AAPOS 2008, 12: $72-76$.

6 Jackson S, Harrad RA, Morris M, Rumsey N. The psychosocial benefits of corrective surgery for adults with strabismus. Br J Ophthalmol 2006; 90: 883-888.

7 Durnian JM, Owen M, Marsh IB. The psychosocial aspects of strabismus; correlation between the AS-20 and DAS59 quality-of-life questionnaires. J AAPOS 2009; 13: 477-480.

8 McKenzie JA, Capo JA, Nusz KJ, Diehl NN, Mohney BG. Prevalence and sex differences of psychiatric disorders in young adults who had intermittent exotropia as children. Arch Ophthalmol 2009; 127: 743-747.
9 Durnian JM, Noonan CP, Marsh IB. The psychosocial impact of adult strabismus: a review. Br J Ophthalmol 2011; 95: 450-453.

10 Hatt SR, Leske DA, Bradley EA, Cole SR, Holmes JM. Development of a quality-of-life questionnaire for adults with strabismus. Ophthalmology 2009; 116: 139-144.

11 Hatt SR, Leske DA, Bradley EA, Cole SR, Holmes JM. Comparison of quality-of-life instruments in adults with strabismus. Am J Ophthalmol 2009; 148: 558-562.

12 Leske DA, Hatt SR, Holmes JM. Test-retest reliability of health-related quality-of-life questionnaires in adults with strabismus. Am J Ophthalmol 2010; 149: 672-676.

13 Hatt SR, Leske DA, Holmes JM. Responsiveness of healthrelated quality-of-life questionnaires in adults undergoing Strabismus surgery. Ophthalmology 2010; 117(12): 2322-2328.

14 Hatt SR, Leske DA, Liebermann L, Holmes JM. Changes in health-related quality of life 1 year following strabismus surgery. Am J Ophthalmol 2012; 153(4): 614-619.

15 Menon V, Saha J, Tandon R, Mehta M, Khokhar S. Study of the psychosocial aspects of strabismus. J Pediatr Ophthalmol Strabismus 2002; 39: 203-208.

16 Durnian JM, Owen ME, Baddon AC, Noonan CP, Marsh IB. The psychosocial effects of strabismus: effect of patient demographics on the AS-20 score. J AAPOS 2010; 14(6): 469-471.

17 Uretmen O, Egrilmez S, Kose S, Pamukçu K, Akkin C, Palamar $\mathrm{M}$ et al. Negative social bias against children with strabismus. Acta Ophthalmol Scand 2003; 81: 138-142.

18 Olitsky SE, Sudesh S, Graziano A, Hamblen J, Brooks SE, Shaha $\mathrm{SH}$ et al. The negative psychosocial impact of strabismus in adults. I AAPOS 1999; 3: 209-211.

19 Coats D, Paysse E, Towler A, Dipboye R. Impact of large angle horizontal strabismus on ability to obtain employment. Ophthalmology 2000; 107: 402-405.

20 Goff M, Suhr A, Ward J, Croley JK, O'Hara MA. Effect of adult strabismus on ratings of official US army photographs. J AAPOS 2006; 10: 400-403.

21 Leske DA, Hatt SR, Liebermann L, Holmes JM. Evaluation of the Adult Strabismus-20 (AS-20) questionnaire using Rasch analysis. Invest Ophthalmol Vis Sci 2012; 53: 2630-2639. 